\title{
Speeds of Chemical Reactions in Biological Processes
}

$\mathrm{S}^{\mathrm{E}}$ VERAL interesting points were raised at the discussion on June 14 at the Royal Society on methods of measuring and factors determining the speed of chemical reactions. The discussion, which was opened by Prof. A. V. Hill, had as its main objective the exploration of methods suitable for attack on biological problems.

Several of the difficulties in such work are now generally recognised; we must note, for example, that physical methods of attack are likely to prove more fruitful than chemical methods, since there are few reagents that do not disturb in some manner the complicated series of reactions proceeding in living matter. Again, in the chemical laboratory it is customary to restrict one's attention to reactions in solution which proceed at speeds conveniently measurable. In biological systems, it is the reactions which are predetermined and their velocities have to be measured. Whilst a half-life of some ten seconds after mixing the reactants is almost the limit of accurate measurement by the usual methods, many biologically important reactions proceed much more rapidly. Prof. H. Hartridge and Dr. F. J. W. Roughton showed how, by means of specially designed mixing chambers, using liquids at high pressures and optical or electrical examination of the mixed liquids in flow, reaction velocitios having a half-life of as small as $1 / 4,000$ sec. could be determined. An extension of this method by Mr. G. Millikan involving a photoelectric cell permits of a greater degree of sensitivity and the elaboration of a micromethod.

The modern extension of the kinetic theory of reactions to complicated molecules is bringing into prominence the importance of what is termed the steric factor; thus, a very large molecule undergoing reaction at one of its constituent groups may be regarded as only potentially active over a relatively small fraction of its total area. These steric factors may play an important part in biological reactions in two somewhat dissimilar fields. We find that steric factors already intrude in such comparatively simple homogeneous reactions as the addition of hydrogen to, say, the double bond in propylene, and may anticipate that there is indeed a very large steric factor in the reactions of, say, oxygen with hæmoglobin. It will be interesting to examine from this point of view the results obtained from measurement of the reactions of the less common and extremely large molecule biological pigments, the molecular weights of which, as determined by the super centrifuge, run into the millions.

Again, numerous biological processes occur at phase boundaries or interfaces, and such reactions possess several interesting peculiarities which are well worth extended investigation. Prof. H. Freundlich pointed out that whilst surface catalysis is a relatively common phenomenon, there are cases in which retardation of a chemical reaction can be brought about by a simple extension of surface; he cited as an example the reaction :

$$
\mathrm{H}_{2} \stackrel{\mathrm{C}}{\mathrm{Cr}} \cdot \mathrm{CH}_{2} \cdot \mathrm{NH}_{2} \underset{\mathrm{HBr}}{\stackrel{\mathrm{NaOH}}{\rightleftarrows}} !_{\mathrm{CH}_{2}}^{\mathrm{CH}_{2}}>\mathrm{NH}+\mathrm{HBr} \text {, }
$$

which is retarded by charcoal in alkaline solution. It is difficult to decide whether the adsorbed mole- cules are firmly adsorbed and so removed from the solution, to which the reaction is confined, or whether reaction is proceeding both in solution and in the adsorbed layer, but in the latter, which may be regarded as an organic medium, the reaction proceeds much more slowly.

In the case of monolayers at fluid interfaces, it is a simple matter to contract or extend the area per molecule by means of a Langmuir trough, and it is possible to examine reaction rates in monolayers by determination of the rate of change in the phase boundary potential. As has been shown in the present writer's laboratory at Cambridge, the velocity constants of numerous reactions in monolayers may be altered to a marked extent by extension or contraction of the film. Thus there is remarkable decrease in the rate of oxidation (by dilute permanganate solution) of the double bonds in a monolayer of oleic acid on suitable compression of the film; or, to suggest a possible analogy to stretched muscle, there is an increased rate of oxidation on extension of the film. The action of enzymes on monolayers of reactants, for example, lecithinase on films of lecithin, is similarly sensitive to an alteration in the molecular concentration or steric factor, which in these cases can be controlled at will.

In addition to the steric factor, the energy of activation plays a dominant part in determining the rate of chemical action, and it is still a matter of speculation as to the accuracy of assessing the magnitudes of the individual energies of activation in the complex chain of biochemical processes. Prof. M. Polanyi pointed out that many ionic reactions in solution require energies of activation and that unsuspected reactions may indeed be taking place. $\mathrm{He}$ cited as a typical example the racemisation of optically active halides by negative ions, which reaction may be written :

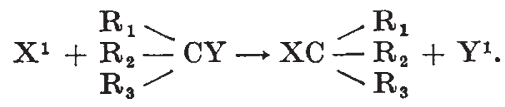

Where $\mathrm{X}^{1}$ and $\mathrm{Y}^{1}$ are identical, that is, $\mathrm{X}^{1}=\mathrm{Y}^{1}$, racemisation can take place without the occurrence of any apparent chemical reaction, although in fact an ion exchange does take place.

Both in chemistry and in physics, a vast number of relative speeds of complicated processes are found to be exponentially temperature dependent, and the mechanisms of these processes are always interpreted on an atomistic hypothesis. Prof. J. B. S. Haldane pointed out that either this inference may not be universally legitimate or a materialistic concept must be given to such curious processes as the subjective measurement of time, for here the logarithm of the relative speed of counting is found to vary inversely as the absolute temperature of the counter, giving a computed energy of activation of 24,000 calories. The speed of bimolecular gas reactions is accelerated by increase of pressure, and the recent experiments carried out by the Imperial Chemical Industries at Northwich have revealed a surprising increase in velocity of many chemical reactions in the liquid phase when suitably high pressures are employed. Prof. Max Cincatel directed attention to this as a possible method of effecting a sudden change in the environment of a living system and examining the 
effect of this change on the various reactions taking place.

Some brief references were also made to the importance of finding a really accurate method for measuring the true permeability rates of extremely thin membranes. Theoretical investigations in this field have already been made by Prof. A. V. Hill, and Dr. F. J. W. Roughton indicated how the stream. ing method could be made applicable to blood cells, thus permitting an examination of the true rates of ingress and egress both of non-electrolytes and of jons.

Eric K. Rideal.

\section{Water Supply}

$I^{\mathrm{T}}$ $\mathrm{T}$ is natural and appropriate that the paramount topic of the drought should find a prominent place in the presidential address of $\mathrm{Mr}$. Councillor Thomas Paris at the annual meeting of the British Waterworks Association (Incorp.) at Edinburgh on June 27. Much of what he had to say respecting the pernicious effects of a shortage of water has been a matter of common experience, but he made the pertinent observation that many of the failures in supply can be traced to procrastination and lack of courage in promoting water schemes. This was more particularly in reference to rural areas where, he emphasised, "the importance of an abundant supply of wholesome water is hardly to be over-estimated" since insufficient or impure water in those areas has wide-reaching effects on public health through milk and foodstuffs produced for general consumption. He alluded to the frequent lack of storage facilities and urged all councils, regional, urban and rural, to take action in the direction of increasing their storage and, where necessary, constructing new waterworks. Another of his points was river pollution, which, he contended, in the national interest must cease. He instanced the case of Edinburgh, where a few years ago there was a turbid stream flowing through the city, "offensive to eye and nostril". The action taken by civic authority has resulted in the transformation of a public nuisance and a menace to health into a "fished water". $\mathrm{He}$ is opposed to the formation of a national water grid, alleging that the argument for such a grid, so far as Scotland is concerned, is without foundation. The question in his view is not one of water shortage, but rather of storage and distribution.

Among the papers contributed to the Conference was one of a particularly timely character on the "Consumption, Misuse and Waste of Water". Mr. John Bowman, the author of the paper, directed attention to the striking difference in the quantities of water supplied per head per day by various authorities. He gave a list of 114 authorities in
England, each supplying a population of more than 50,000 , in which the consumption ranged from 13.00 to 73.45 gallons per head per day. Another list showed that among 27 water authorities in Scotland, the consumption ranged from 34 to 92 gallons per head per day. Commenting on the subject of undue consumption, which might be defined as the use of more water than is necessary, he said : "a person living in a country where. water is scarce may find it possible to perform all his ablutions with one gallon of water per day, and half as much again for culinary and drinking purposes. In civilised countries it would appear that, at least, from 4 to 6 gallons per head must be allowed, where there is no water used for baths or water-closets". Where water is used in addition for the supply of water-closets, it would appear that the lowest figure is about 10 gallons per head. Much depends on the class of property. Houses of the residential class have a higher per capita consumption than small tenements.

Mr. Bowman went on to ask the question: What is to be regarded as the future requirements for ordinary domestic consumption? He gave it as his opinion that within the next twenty years at least 20 gallons per head per day would have to be provided for the increased use of baths. The requirements per head per day would then be in the region of 50 gallons. Perhaps forty years from now a consumption of 80 gallons might be considered possible. In American towns 80 gallons per head is looked upon as a normal consumption. Dealing with the question of waste, which he attributed largely to defective fittings, he stated that a good deal of it might be eliminated by the installation of heavy service piping and good fittings. Useful work, he thought, might be done in educating the householder in the avoidance of waste due to faulty fittings and in getting him to see that taps were left properly turned off and to use water without undue consumption.

\section{Fish Preservation in Trawlers}

$\mathrm{W}$ ITH the introduction of steam-driven vessels - somewhere about the year 1870-the great development of the present long-distance, deep-sea trawling industry became possible. But the industry's present greatness is not due to steam alone. Had not the practice of stowing the catch in crushed ice been also introduced about the same time, the bringing back of fish in a saleable condition from far distant grounds would have been impossible even for large and powerful steamers unaffected by the vagaries of wind-propulsion.

In recent years many experiments have been made in an endeavour to evolve and perfect a more satisfactory method of preserving fish at sea. In spite of every effort towards improvement, however, the stowage of trawled fish in crushed ice is still the general practice, notwithstanding its very serious limitations.

The preservative effect of crushed ice is two-fold. By lowering the temperature of the fish tissues, changes due to autolysis are slowed down. This lowering of temperature also slows down the rates at which the bacteria of decay grow and multiply; but stowage in crushed ice alone cannot inhibit their activities completely.

Bacteria of decay are present on the fish when caught, but only in negligible numbers. As at present handled on board ship after capture, however, the fish become very heavily infected with these organisms. As a result of this severe infection, storage 